\title{
Digital Culture for Optimization
}

\author{
Samir Al-Azri
}

\begin{abstract}
Humanity proceeds along the path of hybridization with the technological tool. The centre of gravity of the creative process moves within the computational man-tool ecosystem, with capital consequences: the instrument, like an "organ", guides our way of thinking, designing, and planning. In this context two aspects of the process of architectural design will be explored in this chapter which requires more consideration from a methodological perspective: design optimization and architectural simulation. The optimization stage is related to the process of realizing the objective of architecture, inherently prescribing the outcome. Representation and simulation, on the other hand, are important as a tie between the concept (or an idea of the architect) and the materialization of that concept, that is, a link between the sensory and non-sensory. Both aspects are important but highlight a different side of the process.
\end{abstract}

Keywords Topology optimization · Digital simulation $\cdot$ Computation in nature $\cdot$ Adaptive topology

\section{Introduction}

Architecture, like music or other forms of creative works, is based on the experience that is evoked by the sensory capabilities of the user. Unlike music which disappears with the sound, the building has the power to enforce its dominance by its permanence (Drewniak 2009). Not only does the building not disappear, but it has the opportunity of lasting and being experienced by different generations.

Architecture is relative by nature. It is undefined, unmeasurable, and is dependent on the perspective of the viewer. A simple task of trying to research a definition would yield countless philosophical terms and quotes from renown architects, all providing their own account or perspective on the matter. Such subjectivism has

\footnotetext{
S. Al-Azri (凶)

Architecture, Built Environment and Construction Engineering-ABC Department, Politecnico Di Milano, Milan, Italy

e-mail: samir.alazri@polimi.it

B. Daniotti et al. (eds.), Digital Transformation of the Design, Construction and Management Processes of the Built Environment, Research for Development, https://doi.org/10.1007/978-3-030-33570-0_12
} 
created a professional community that is dispersed more than it is united. The meaning of architecture spans a large spectrum from Hassan Fathy's "architecture of the poor" to Frank Gehry's extravaganza. Within this fragmentation, the main discourse about architecture today examines whether it is an art form, a process/practice or a final product (Fisher 2015). Architectural practice and research in recent years have touched upon completely new fields of study. The technological advancements and scientific discoveries from different fields drive more architectural processes. Focusing on the process/practice discussion, it is imperative to consider the role of the advanced technologies we have available today and how the design culture is shaped by the digital revolution.

In this context two aspects of the process of architectural design will be explored in this chapter which requires more consideration from a methodological perspective: design optimization and architectural simulation. The optimization stage is related to the process of realizing the objective of architecture, inherently prescribing the outcome. Representation and simulation, on the other hand, are important as a tie between the concept (or an idea of the architect) and the materialization of that concept, that is, a link between the sensory and non-sensory. Both aspects are important but highlight a different side of the process.

\section{Optimization}

Optimization is the process of finding the best and optimal solution to a given problem-an idea that can be traced back to the Ancient Greeks, but only gained momentum in application with Newton's development of modern calculus (Kiranyaz 2014). A renowned optimization problem is the travelling salesman problem, where a salesman must visit all the cities in the United States but should figure out the path that would minimize the travelling distance without visiting the same city twice. This problem is still in use today to benchmark optimization algorithms. The reasoning of optimization is based on the existence of an optimum solution in which the designer can strive to achieve. From that premise, the designer should be able to explore the large solution space to identify that one ideal solution. This process is usually in the later design phases and involves committing an extensive amount of resources to optimize it and usually requires expertise from the designer.

Nature, as we know it, is the result of billions of years of development and adaptation. It features panoply of organisms that inherit this long process of evolution including ourselves. However, as Alan Watts contemplates in his book "The book on the taboo against knowing who you are", we as humans suffer from a hallucination, from a false and distorted sensation of our own existence as living organisms. We have been brought up to believe that we came into this world rather than we came out of it. This simple ideological twist alone might draw our very distinction from every other organism. When nature was conceived as something alien and hostile, we decided to conquer it rather than cooperate with it (Watts 1973). We marked a unique geological era which is called by the new science "the era of Anthropocene". 
It seems that we have been successful at conquering the world but at the cost of jeopardizing our own existence.

Despite different outlooks on architecture, finding solutions for the conditions brought about by ourselves, now loom over as an oppressive imperative for the future practice (Luebkeman 2015). Architects can no longer indulge themselves with designing beautiful buildings without considering their environmental impacts. For the first time, we can sense the urgency of proactively participating in more sustainable practice. We are left with no other choice other than cooperating with nature to guarantee our survival as a species. More attention has been drawn to natural sciences and its subdisciplines in recent years in response. The most cutting-edge institutions/studios around the globe are increasingly committing themselves to the study of nature and its potential pertinence to architecture.

A new "design culture" as mentioned by Neri Oxman is emerging, thanks to the advancements of technology. It is distinguished by the underlying approach to the very act of creating. Products and buildings, for the most part of the history of craftsmanship, have been created by assembling different parts. However, now at the intersection of computational design, additive manufacturing, materials engineering and synthetic biology, we can grow products and even our buildings just as nature. Instead of having distinct parts delivering different purposes, the output of the new process is a product with varying properties, gradually transitioning its functionality over different areas exactly the same way that the trunk of a tree transitions into its branches, leaves and so forth (Oxman 2012).

The "Silk Pavilion" project developed by the Mediated Matter Group at MIT Media Lab capitalizes on the inherent intelligence of silkworm to optimally produce silk fibres of variable properties, according to the functions needed. These fibres are optimized for a wide range of different conditions including, but not limited to, mechanical properties such as strength and toughness. This research explores the possibility of merging digital and biological fabrication to deliver a holistic and sustainable design approach in the production of non-woven fibre-based constructions (Oxman et al. 2014).

In another example, a project carried out by the students of The Bartlett School of Architecture explores the embedded computational capabilities of Slime Mold or Physarum polycephalum for generating urban design proposals for Liwa Oasis in UAE. Physarum polycephalum is a single biological cell with countless cell nuclei. This brainless organism can stretch itself to any shape in multiple branches called plasmodium searching for resources. Upon facing a source of food, it leaves traces as a memory, otherwise eventually fading away in the absence of food. The outcome of this rather heuristic process is an optimized network distributed between the sources. This lends a sophisticated network optimization model to be adopted for scenarios such as Liwa where future developments heavily rely on the informed use of the existing resources (UCL 2015).

There is a fundamental issue in the optimized design process, which is the emphasis on a certain design objective and disregarding the rest. For an optimization process to converge into a single solution, a certain function would have to be minimized or maximized. This implies that all significant design outcomes are quantitative. An 
example would be the optimization of the energy consumed in a building. A building, however, is more than the measure of its performative quantifiable properties (the sum is always larger than the parts). However, there are many other factors that render a building design meaningful. This goal-oriented approach has given rise to many factions each concerned with what they consider as the most important aspect of a building design. Performance-driven designs, for example, emerged recently as a response to the energy crisis and a solution to climate change. It is not to say that their goals are not legitimate, however, it can be argued that other design characteristics are just as important.

Genetic algorithms are prominent in the design optimization scene today. However, we can apply the concept of "survival of the fittest" to the buildings as well. Only the outstanding buildings were worth being preserved; the rest were demolished with time. The architects of the past surely built many buildings that were not considered worth keeping or documenting, and that was probably most of the cases. Nevertheless, we are accustomed to looking back and applauding past generations for their work, neglecting the fact that they made mistakes just the way we do today. Architecture as a concept is going through an optimization process within itself and we are converging to a better built environment with time.

The ecological paradigm and computational design thinking in architecture have led to novel methodologies. Finding inspirations from nature, which is commonly given the term "bio-mimicry", has a long history in the architectural practice. However, it has been for the most part, limited to mimicry of the forms and shapes. This approach, despite exceptions of good examples, is a rather thin abstraction of what nature can offer in terms of efficiency and potential solutions for complex problems.

On the positive side, more architects are either involving themselves with in-depth study of natural processes or resort to close collaboration of specialists in other fields including biology and genetics, finding solutions for future challenges. An increasing number of inter-disciplinary studios are emerging. There is a shift in the perception of nature from a mere source of aesthetical inspiration, towards a repertoire of the intelligence which is developed through billions of years of evolution (Pawlyn 2011). The most cutting-edge institutions and research labs are striving to decipher complex natural systems and implementing their knowledge of their processes. These attempts include translating the optimized behaviours of particular organisms into tailor-made solutions for particular design problems, modifying certain natural organisms to find optimum functionality for certain purposes or directly applying the organisms within the design process.

\section{Simulation in Architecture}

Architecture is realized in a dimension between what we envision and what exists. Considering the mind as the source of concepts and the place where ideas are processed, and the building as the final product, there lies a medium in between through 
Ideas

Design Thinking

$3 \mathrm{D}$ conceptualization

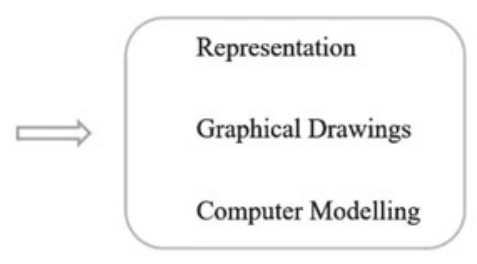

Physical reality

Built matter

Material in

Euclidean space

Fig. 1 The medium between concept and realization

which information needs to be communicated. This intermediate bridge between the two realities was previously referred to as architectural representation (Fig. 1).

Representation in architecture is the accumulation of information found in geometry. The representation can take many forms, from drawings to physical models, and the amount of information determinable depends on the viewing perspective. A computer model, for instance, can be rotated and viewed from an infinite number of positions. However, the information transferred through the screen is that of a still image at a specific timeframe. Therefore, we can think of representation as the collection of information from all perspectives, and hence all images.

The idea of images, and information stored within, incites one to question the understanding of geometry. Starting with the Greeks and the introduction of the Euclidean geometry, the ability to deduct measures from drawings constantly puzzled the mathematicians. This was only rationalized during the Renaissance period with the works on perspectives, making it a revolutionary period for architectural graphics. However, curves remained the most difficult objects to represent, and circles were used to estimate the curvature at different points of a curve.

There are no solids in the universe. There's not even a suggestion of a solid. There are no absolute continuums. There are no surfaces. There are no straight lines.-Buckminster Fuller

Simple geometries can be transformed and rationalized into complex geometries through morphing, be it addition or subtraction. Similar works can be observed in the works of Philibert Delorme and his shell models. This approach of understanding geometry raises the question of the reality of the models and objects we are building. It is understood that a curve is nothing but an estimation created from many smaller circles that are connected through their tangent vectors, and no perfect sphere can be created. On the other hand, we rest assured that we can build a structure that is perfectly straight and proclaiming that is. The tools used are levels that check the altitude difference between two points, but since the earth is not flat, how straight are these "straight line"? In our microscale compared to the world, such an approximation is accepted. However, the models and calculations used to ensure a structure is stable, are perfect geometries. Is this an acceptable deduction? Or are we dependent on the confidence of our experimental realism?

If process drives outcome, we may not know where we are going, but we will know we want to be there-Bruce Mau. 
It is suggested by Maurice Conti in his Ted Talk that we are on the cusp of a new age of our activities as a species. After the four major eras that define how we have been working, namely, hunter-gatherer, agricultural, industrial and digital age, we are now at the dawn of a new era called "augmented age". For the first time in history, the tools developed by humankind will augment it through its daily actions. Unlike how tools worked in the past, merely in a passive form that needed direct exertion of intention and authority from its user, new tools are being developed to actively contribute and even think (Conti 2017).

Simulation has always been a key element in the process of design. Every design undergoes some sort of simulation, even at the most elementary level. An architect will simulate, at least in a mental simulation, the way the building occupants will interact in a space. This simulation will be driven by certain design drivers or objectives, while the constraints will depend on gained knowledge through experience, maybe construction techniques or local codes. This simulation is limited to the designers computing capabilities and has been historically outsourced to physical models to try and comprehend the complexity at hand. Great examples include working with strings and weights, and soap films, by Gaudi and Frei Otto, respectively.

With the advancement of computing powers in the digital age, the interaction between human and technology evolved to an unprecedented level. Technology has become an extension of human activities, leveraging their abilities in all aspects. Transhumanism is a common term now from magnetic implants to augmented reality. In the design field, simulations have come a long way as technological tools. Mathematical algorithms have been developed that make digital simulations of physical phenomenon's accessible to designers and engineers. Interestingly, the design process did not adopt these tools as design drivers at first and instead used these models mostly in the advanced stages to validate or analyze the performance of a proposal. This shift allocated the design solution generation responsibilities to the designer and limited the simulation process to selection or enhancements. Naturally, the solution space explored was limited and the designs produced by human capabilities were streamlined to compliment a familiar manufacturing process.

The challenges on the horizon are increasingly growing complex for humans to handle. The problems lying ahead such as climate change are far more complex for us to analyze and organize by ourselves. However, our capabilities are being augmented by emerging technologies. The most advanced computers lend us unprecedented powers of analyzing millions of data points only in a matter of seconds (Conti 2017). Novel manufacturing technologies are capable of producing almost anything. Simulation in this regard finds a vital role. And how we go about designing as architects is subject to fundamental disruption.

Simulation as a by-product of the advancements of technologies has been turning into an active collaborator for designers. It is not only a model to validate the feasibility of a design per se, but also a contribution to an informed design process. The computational simulations shed light on completely new facets of our propositions, giving us real-time models of the consequences of our decisions. Therefore, the design outcomes as mentioned by Mau will somewhat become unpredictable but in a rather positive way. 
As previously mentioned, more designers and researchers are turning to nature to find solutions for their complex design problems. In an attempt of adopting the ingenuity of many different organisms, the research can sometimes lead to a team of computational scientists trying to develop algorithms or models that simulate the favoured behaviours and patterns being played out by the particular organisms. These models will, later on, be exploited as simulation tools that will allow them to implement the intelligence of nature into their design process. For example, in the case of Liwa Oasis, in order to perform the same network optimization process at the local scale, the biological algorithm of slime mould was digitally simulated (UCL 2015). In the case of "The Silk Pavilion", in order to understand the structural complexity of a cocoon, advanced imaging techniques were carried out using magnometer motion sensing to capture the movements and the patterns of the silkworm during the production of the cocoon. The silkworm motion-tracked during the three days yielded a point cloud model with high levels of precision to be investigated (Oxman et al. 2014). Eventually, the investigations led to a more informed design of the silk scaffold.

The simulations provided us with means of understanding and visualizing forces and energy. This process allowed us to build towers and skyscrapers cladded with glass that are consuming significantly more energy for heating and cooling. Consequently, a climatic crisis evolved and we are currently trying to simulate our way out of this calamity. How much of the technology contributed to today's environmental challenges and can we trust it not to create more? Or will it be the saviour and enhance our well being?

\section{Case Study: Adaptive Topology}

To examine the influence of the digital tools on designers, an experiment was conducted in the form of a workshop. A group of 10 students were introduced to a new process of simulation in the form of topology optimization. This optimization process would allow their designs to be remodelled in a topology that reduces material while complying structurally. The students did not have prior knowledge of the process and were introduced for this first time during the workshop.

The design task in hand was to design a chair for the Salone Del Mobile 2018. Initially, the students were given the freedom of interpreting their own design processes and the only given requirement was that the chair was to be fabricated through additive manufacturing. Upon completion of their first concept designs, the students were then introduced to topology optimization and the available simulation tools; Ameba through Rhino/Grasshopper and Inspire by Altair. The students were then given the options of either starting the design from scratch and using the new simulation tool as a generative design process, or remodel their existing designs to reduce the amount of material needed for fabrication. 
The results revealed that all the students attempted improving their designs with the topology optimization process, but none steered away from their initial concept/ideas. Reducing the amount of material was a sustainable task, which they agreed was necessary and were willing to undertake. However, they did not compromise on their initial form and design (Fig. 2). In addition, none of the participants was tempted to restart their design process with the new simulation tools. This observation questions the idea of a design agency in the new digital era and focuses on the designer's continuous demand for autonomy. The continuous disruptions caused by explosive innovations in different fields raise the question of authority in design. Designers may well have to adapt themselves to the present conditions that necessitate collaboration from many different sources. Despite the predictions about the future, arguing that technology would take over in different sectors, we might as well hope for better future scenarios that will be played out by the productive collaboration of humans, nature and technology.
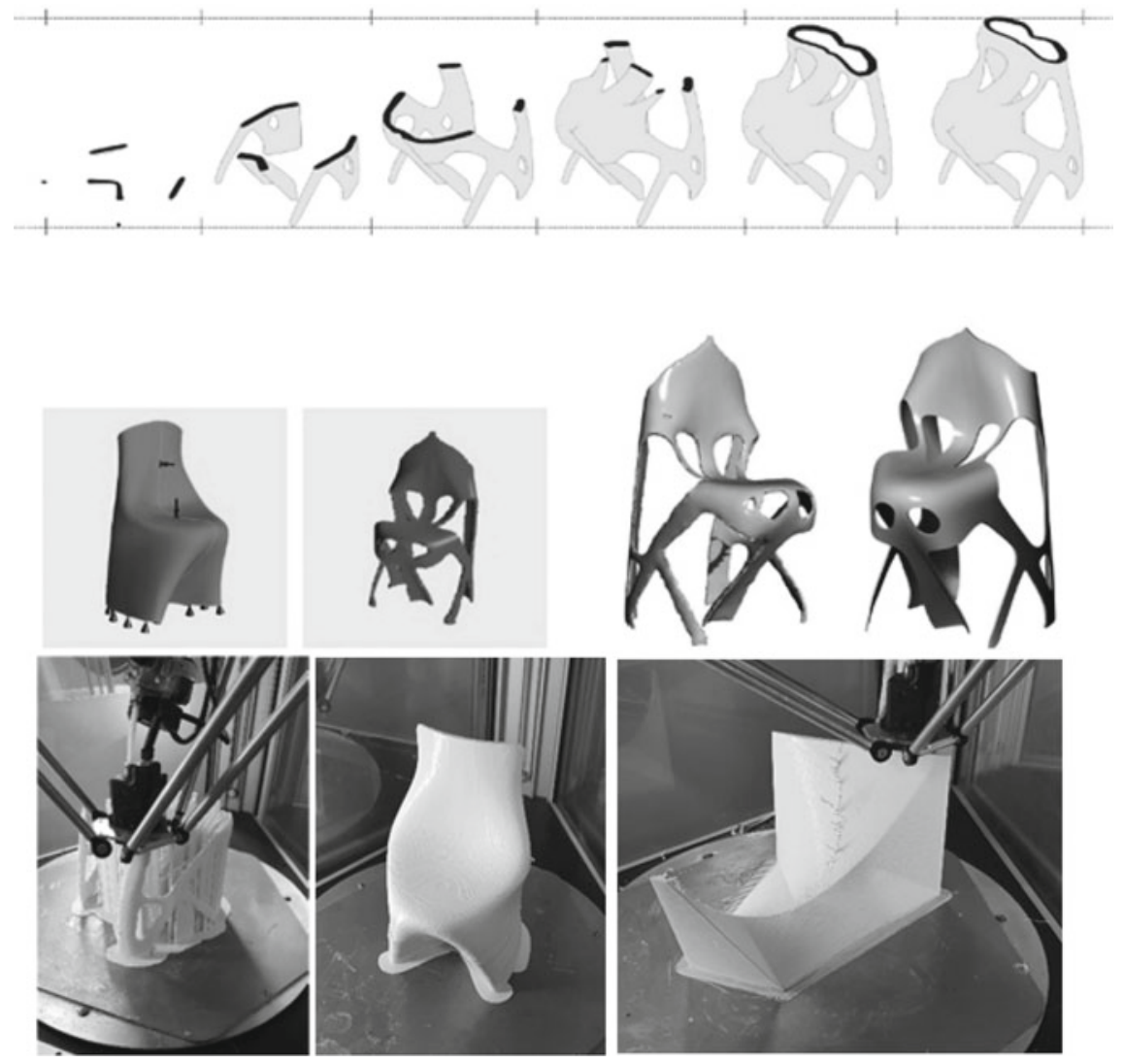

Fig. 2 Design and production of "Adaptive Topologies" workshop at Politecnico di Milano. Image Credits Iris Koni, Elena Manandise, Zeynep Kalaycioglu 


\section{References}

Architectural Record (n.d.) Accessed from https://www.architecturalrecord.com/ext/resources/ archives/features/critique/2012/images/1204-Commentary-slideshow/Perspective-Drawing-3. jpg

Conti M (2017) The incredible inventions of intuitive AI. [video] Available at: https://www.ted. com/talks/maurice_conti_the_incredible_inventions_of_intuitive_ai/discussion?langua

Drewniak T (2009) Philosophy of architecture and architectonics of philosophy. Filosofía UIS 8(2):11-31

Fisher S (2015) Philosophy of architecture. Accessed from Stanford Encyclopedia of Philosophy: https://plato.stanford.edu/entries/architecture/

Kiranyaz S (2014) Multidimensional particle swarm optimization for machine learning and pattern recognition. Springer, Berlin

Luebkeman C (2015) 2050: designing our tomorrow. Wiley, New York

Mumford L (2015) Mass-production and the modern house. Accessed from Architectural Record: https://www.architecturalrecord.com/ext/resources/archives/inTheCause/onTheState/ 0311mumford.pdf

Oxman N (2012) Material computation. Wiley, New York

Oxman N et al (2014). Silk pavilion: a case study. In: Fiber-based digital fabrication. gta Verlag

Pawlyn M. (2011). Biomimicry. In: Architecture. RIBA Publishing

Stasinopoulos TN (2015) The need for architectural materialism. Plea, Bologna

The Bartlett School of Architecture, UCL. (2015). MArch Urban Design (UD) 2015. The Bartlett School of Architecture, UCL

Watts A (1973) The book; on the taboo against knowing who you are. Vintage Books

Open Access This chapter is licensed under the terms of the Creative Commons Attribution 4.0 International License (http://creativecommons.org/licenses/by/4.0/), which permits use, sharing, adaptation, distribution and reproduction in any medium or format, as long as you give appropriate credit to the original author(s) and the source, provide a link to the Creative Commons license and indicate if changes were made.

The images or other third party material in this chapter are included in the chapter's Creative Commons license, unless indicated otherwise in a credit line to the material. If material is not included in the chapter's Creative Commons license and your intended use is not permitted by statutory regulation or exceeds the permitted use, you will need to obtain permission directly from the copyright holder.

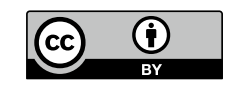

